\title{
EQUILÍBRIO ÁCIDO-BASE
}

\author{
ACID-BASE BALANCE
}

Paulo Roberto Barbosa Évora', Luis Vicente Garcia²

\begin{abstract}
'Docente da Divisão de Cirurgia Torácica e Cardiovascular do Departamento de Cirurgia e Anatomia; ${ }^{2}$ Docente do Departamento de Ortopedia e Biomecânica. Faculdade de Medicina de Ribeirão Preto - USP

Correspondência: Paulo Roberto Barbosa Évora. Divisão de Cirurgia Torácica e Cardiovascular do Departamento de Cirurgia e Anatomia. Av. Bandeirantes, 3900. 14048-900. Ribeirão Preto / SP. email: prbevora@fmrp.usp.br
\end{abstract}

Évora PRB, Garcia LV. Equilíbrio ácido-base. Medicina (Ribeirão Preto) 2008; 41 (3): 301-11.

RESUMO: É imprescindível o conhecimento do diagnóstico e do tratamento das alterações do equilíbrio ácido-base. Nessa apresentação são abordados a causa, os efeitos e o tratamento da alcalose e da acidose, respiratória e metabólica.

Descritores: Equilíbrio Ácido-base. Alcalose. Acidose.

\section{1- CONCEITOS FISIOLÓgICOS FUNDA- MENTAIS}

A avaliação do estado ácido-básico do sangue é feita na grande maioria dos doentes que são atendidos em Unidades de Terapia Intensiva, qualquer que seja a doença de base; essa avaliação é fundamental, pois, além dos desvios do equilíbrio ácido-base (EAB) propriamente dito, pode fornecer dados sobre a função respiratória e sobre as condições de perfusão tecidual.

Os sistemas orgânicos enfrentam dois desafios básicos para a manutenção do EAB. O primeiro é a disposição da cota fixa de ácidos ingerida na dieta diária; o segundo é o destino dado ao $\mathrm{CO}_{2}$ gerado como produto final do metabolismo. Para manter o $\mathrm{pH}$ em limites compatíveis com os processos vitais o organismo lança mão de uma série de mecanismos bioquímicos, com destaque para o papel desempenhado pelo chamado sistema-tampão. O sistema tampão do organismo apresenta quatro grandes componentes: bicarbonato/ácido carbônico, proteínatos/proteínas, fosfatos monoácidos/fosfatos biácidos e hemoglobinato/he- moglobina. As substâncias-tampões são responsáveis minimizar as alterações do $\mathrm{pH}$ decorrentes da adição ou subtração de íons $\mathrm{H}^{+}$. Com a queda do $\mathrm{pH}$ da solução, estas substâncias aceitam os íons $\mathrm{H}^{+}$para entregá los novamente quando aumenta o $\mathrm{pH}$; desta maneira agem contra as modificações abruptas da reação. Entre os tampões do espaço extracelular o bicarbonato e as proteínas plasmáticas desempenham papel relevante, enquanto a hemoglobina e os fosfatos estão em primeiro plano no compartimento intracelular.

Graças a estes sistemas de tamponamento, pequenas alterações do EAB manifestam se por um deslocamento do equilíbrio da reação dos tampões com atenuação de modificações significativas da concentração dos íons $\mathrm{H}^{+}$livres ou do pH. O tampão bicarbonato é o mais importante. ${ }^{1,2}$

$$
\mathrm{CO}_{2}+\mathrm{H}_{2} \mathrm{O}^{-}>\mathrm{H}_{2} \mathrm{CO}_{3}^{-}>\mathrm{H}^{+}+\mathrm{HCO}_{3}^{-}
$$

A partir desta reação química é possível deduzir a equação de Henderson Hasselbach, fundamental para o entendimento do equilíbrio ácido base: 


$$
\mathrm{pH}=\mathrm{pK}+\log \frac{\left[\mathrm{HCO}_{3}^{-}\right]}{\mathrm{H}_{2} \mathrm{CO}_{3}}
$$

O ácido carbônico pode ser representado pelo produto da constante 0,03 pela pressão parcial de $\mathrm{CO}_{2}$ do sangue arterial.

$$
\mathrm{pH}=\mathrm{pK}+\log \frac{\left[\mathrm{HCO}_{3}^{-}\right]}{0,03 \times \mathrm{paCO}_{2}}
$$

Surge no denominador da equação um gás que expressa a ventilação. Assim é possível concluir que a manutenção do $\mathrm{pH}$ depende fundamentalmente da função renal (numerador da equação) e da função respiratória (denominador da equação).

\section{Função renal \\ $\mathrm{pH}=\frac{\text { (componente não respiratório ou metabólico) }}{\text { Função respiratória (componente respiratório) }}$}

O termo "metabólico" é um erro consagrado pelo uso, uma vez que ambos os componentes da equação de Henderson Hasselbach envolvem um processo metabólico.

Considerando se estes conceitos, os mecanismos de excreção do $\mathrm{H}^{+}$podem assim ser resumidos: ${ }^{2}$

- A excreção pulmonar do íon $\mathrm{H}^{+}$é indireta. O pulmão excreta somente substâncias voláteis, isto é, substância que podem ser convertidas em gases.

- A excreção renal não apresenta esta limitação, sendo coadjuvada pelas bases tampões.

- A média de excreção de $\mathrm{CO}_{2}$ pelos pulmões pode ser mudada rapidamente em qualquer direção por alterações apropriadas da ventilação alveolar, porém a hipoventilação para poupar $\mathrm{H}^{+}$não é um mecanismo tão eficiente quanto a hiperventilação que elimina este mesmo íon de maneira eficiente no início de um

acidótico.

- A função renal pela excreção de $\mathrm{H}^{+}$e eletrólitos influencia no estado ácido base do líquido extracelular (LEC), porém um período de horas é necessário para que esta influência seja significante.

Um outro detalhe interessante da fisiologia do EAB são as evidências de que o organismo animal, na sua evolução, adquiriu mecanismos naturais de defesa contra a acidose mais eficientes do que os mecanismos contra a alcalose:
- Características próprias da função renal que elimina o $\mathrm{H}^{+}$e reabsorve o $\mathrm{HCO}_{\overline{3}}$.

- Curva da dissociação da hemoglobina (Hb): na acidose a curva desvia se para a direita diminuindo a afinidade da $\mathrm{Hb}$ pelo oxigênio e na alcalose desvia se para a esquerda, aumentando a afinidade.

- A hiperventilação com alcalose respiratória, desviando a reação do $\mathrm{CO}_{2}$ com a água para a esquerda, é um mecanismo natural de compensação da acidose metabólica, que pode ocorrer normalmente, por exemplo, no exercício físico. A ocorrência do fenômeno inverso é possível na teoria, mas a depressão respiratória produzindo uma acidose respiratória para compensar uma alcalose metabólica só ocorreria com uma diferença de base $>+40$, uma situação praticamente impossível; isto reflete uma maior dificuldade do tratamento da alcalose metabólica, como se observa na prática diária da terapia intensiva.

É importante conceituar reserva alcalina e "base excess" (BE). A reserva alcalina (capacidade de ligação de $\mathrm{CO}_{2}$ ) é a quantidade total de $\mathrm{CO}_{2}$ de uma amostra de plasma separada anaerobicamente após equilibrá-la com $40 \mathrm{mmHg}$ de $\mathrm{pCO}_{2}$ à temperatura ambiente (usa se de preferência o bicarbonato padrão em $\mathrm{mMol} / l$ (escola americana capitaneada por DAVENPORT).

$\mathrm{O}$ excesso de bases, ou diferença de bases, é igual à concentração de bases do sangue em $\mathrm{mEq} / \mathrm{l}$, tituladas por um ácido forte a um $\mathrm{pH}$ de 7,40, com uma $\mathrm{pCO}_{2}$ de $40 \mathrm{mmHg}$ e à temperatura de $37^{\circ} \mathrm{C}$ (escola dinamarquesa capitaneada por SIGGAARD ANDERSEN).

Com a crescente simplificação da análise do EAB por aparelhos de alto rendimento, a determinação de parâmetros isolados, como a reserva alcalina, em comparação com um levantamento completo do EAB, passou para um segundo plano.

Existe importante inter relação entre o EAB e o equilíbrio hidroeletrolítico (EHE). ${ }^{2}$

\section{1- Lei da Eletroneutralidade}

Estabelece que a soma das cargas negativas dos ânions deve ser igual à soma das cargas positivas dos cátions. No plasma há $154 \mathrm{mEq} / l$ de cátions e, consequientemente, $154 \mathrm{mEq} / l$ de ânions. Em todas as circunstâncias o sódio responsabiliza se pela maior parte dos equivalentes catiônicos; portanto, o bicarbonato constitui o elo entre o EAB e o EHE, já que faz parte de ambos. Assim, para que se mantenha a eletroneu- 
tralidade, na vigência de queda do bicarbonato, ocorre um aumento do cloreto e vice versa. O entrejogo dos prótons e ânions em acumulação com os componentes normais do soro resulta em padrões de eletrólitos que possibilitam a classificação dos tipos de acidose metabólica. Os prótons consomem as reservas de álcalis, manifestando sua presença sob a forma de hipobicarbonatemia. Como o álcali perdido é substituído por anions ácidos (fosfato, acetoacetato, cloreto, etc.), segue se que qualquer ácido, exceto o HCL, substitui o ânion $\mathrm{HCO}_{\overline{3}}$, facilmente medido, por ânions que não são medidos rotineiramente. A "diferença de ânions" ("anion gap" = DA) reflete o balanço entre o cátion rotineiramente medido $\left(\mathrm{Na}^{+}\right)$e os ânions rotineiramente medidos $\left(\mathrm{CL}^{-}+\mathrm{HCO}_{\overline{3}}\right)$.

$$
\mathrm{DA}=\mathrm{NA}^{+}-\left(\mathrm{CI}^{-}+\mathrm{HCO}_{\overline{3}}^{-}\right)
$$

Como a acidose por HCL efetua uma troca igual de $\mathrm{HCO}_{3}^{-}$perdido por $\mathrm{CI}^{-}$retido, sem alterar necessariamente $\mathrm{o} \mathrm{Na}^{+}$sérico, a DA permanece na faixa normal $(12 \pm 2 \mathrm{mEq} / l)$, dessa maneira definindo uma acidose hiperclorêmica com DA normal. Todas as outras formas de acidose aumentam o "anion gap" ao substituir o $\mathrm{HCO}_{\overline{3}}$ por ânions diversos do $\mathrm{CL}^{-}$. Uma análise crítica da utilidade prática do conceito de "anion gap" demonstra a sua pequena utilização na prática diária. Qual seria a sua real utilidade? Diagnóstico diferencial entre tipos de acidose permitindo pensar em diagnósticos menos comuns? Como índice prognóstico? Uma utilidade real seria o controle de qualidade de dosagens laboratoriais, já que não existe a situação de "anion gap" aumentado, que ocorre só na condição de erros de dosagem. Merecem menção os nomogramas de Gamble para a ilustração e integração entre o EAB e o EHE.

\section{2- Lei da Isosmolaridade}

Determina que a osmolaridade é a mesma nos compartimentos líquiidos do organismo, entre os quais a água passa livremente. Seu valor normal é em torno de $285 \mathrm{mOsm} / l$, e, se o número de partículas dissolvidas aumenta em um compartimento, a água se mobilizará em direção a ele até que um novo equilíbrio da osmolaridade seja estabelecido.

\section{3- Lei Fisiológica do Equilíbrio Ácido base}

Estabelece que o organismo tende a manter o $\mathrm{pH}$ do sangue em torno de um valor normal. Quanto a esta lei, um fato importante é a interação entre $\mathrm{o} \mathrm{K}^{+} \mathrm{e}$ $\mathrm{o} \mathrm{H}^{+}$em relação ao intra e extracelular. No caso de acidose, na tentativa de manter $\mathrm{o} \mathrm{pH}$ do sangue, $\mathrm{o}$ potássio sai da célula com a entrada do $\mathrm{H}^{+}$, ocorrendo o contrário na alcalose, ou seja, saída do $\mathrm{H}^{+}$e entrada do potássio para o compartimento intracelular.

\section{2- DIAGNÓSTICO DOS DISTÚRBIOS DO EAB PELA ANÁLISE GASOMÉTRICA}

Por tratar se de um texto apenas com pretensões didáticas, antes da apresentação das causas dos distúrbios do $\mathrm{EAB}$, que seria a seqüência lógica, a seguir serão apresentados os princípios diagnósticos. Esta inversão tem-se mostrado útil, pois torna fácil a dedução posterior das variadas causas de desequilíbrio ácido base. ${ }^{2}$

O diagnóstico das alterações do EAB é feito pela análise dos valores obtidos pela gasometria sangüínea. Como todo exame laboratorial, existem fatores que podem influenciar seus resultados:

- Em pacientes conscientes, a punção arterial pode resultar em hiperventilação induzida pelo temor ao próprio procedimento.

- A heparina é ácida e ao menos teoricamente pode influenciar os valores do $\mathrm{pH}, \mathrm{pCO}_{2}$ e $\mathrm{pO}_{2}$ em amostras pequenas.

- A presença de leucocitose e grande número de plaquetas reduzirá o valor do $\mathrm{pO}_{2}$, dando a falsa impressão de hipoxemia. Esta queda na $\mathrm{pO}_{2}$ é negligenciável se a amostra é armazenada em gelo e analisada dentro de 1hora; presumivelmente o metabolismo em andamento dos elementos celulares do sangue pode consumir $\mathrm{O}_{2}$ e reduzir a $\mathrm{pO}_{2}$.

- O resfriamento aumenta o $\mathrm{pH}$ e a saturação de oxigênio, e diminui a $\mathrm{pO}_{2}$.

- O halotano aumenta falsamente os valores da $\mathrm{pO}_{2}$, porque o eletrodo para a $\mathrm{pO}_{2}$ no aparelho de gasometria também responde ao halotano.

Ainda sob o ponto de vista da análise gasométrica, alguns pormenores merecem destaque:

- A adoção da escola dinamarquesa (SIGGAARD ANDERSEN) é praticamente uma unanimidade internacional.

- Quando se solicita a gasometria sangüínea, o pH, a $\mathrm{pO}_{2}$ e a pCO $\mathrm{pCa}_{2}$ a obtidos nor medida eletrônica direta. Os valores do $\mathrm{CO}_{2}, \mathrm{HCO}_{\overline{3}}, \mathrm{BE}$, saturacão de oxigênio $\left(\mathrm{Sat}_{\mathrm{O} 2}\right)$ são obtidos por medida indireta mediante leitura no nomograma de Siggaard Andersen. 
- Como os distúrbios envolvem mecanismos renais e respiratórios, o diagnóstico deve ser baseado na gasometria do sangue arterial.

- A amostra venosa não permite a análise da função respiratória e sua colheita está sujeita a erros. Para fins científicos deve, inclusive, ser colhida na artéria pulmonar onde o sangue venoso é misto. Duas informações práticas, porém, podem ser obtidas pela análise da gasometria venosa:

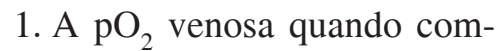
parada com a $\mathrm{pO}_{2}$ arterial dá uma idéia do débito cardíaco (diferença arteriovenosa grande com a $\mathrm{pO}_{2}$ venosa baixa significa baixo débito, com os tecidos extraindo muito o oxigênio da hemoglobina pelo fluxo lento, sendo esta uma situação ainda favorável para se tentar a reversão de um estado de choque).

2. A diferença arteriovenosa pequena com progressivo aumento da $\mathrm{pO}_{2}$ venosa indica um "shunt" sistêmico, isto é, um agravamento das trocas teciduais. Portanto, o principal dado fornecido pela gasometria venosa é a $\mathrm{pO}_{2}$.

O diagnóstico dos distúrbios do $\mathrm{EAB}$ deve, assim, embasar-se nos valores da gasometria arterial. É importante conhecer os valores normais dos parâmetros da gasometria arterial (Quadro 1).

Pela gasometria arterial é possível o diagnóstico dos desvios do componente respiratório $\left(\mathrm{O}_{2} /\right.$ oxige-

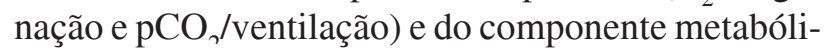
co $\left(\mathrm{BE} \mathrm{e} \mathrm{HCO}_{\overline{3}}\right)$ (Figura 1).

$$
\begin{array}{ccc}
\text { Quadro 1: Valores normais da gasometria arterial } \\
\hline \mathrm{pH} & =7,35-7,45 & \mathrm{CO}_{2}=23-27 \mathrm{mmol} / l \\
\mathrm{pO}_{2}=70-90 \mathrm{mmHg} & \mathrm{HCO}_{\overline{3}}=22-26 \mathrm{mEq} / l \\
\mathrm{pCO}_{2}=35-45 \mathrm{mmHg} & \mathrm{BE}=-3,5 \mathrm{a}+3,5 \mathrm{mEq} / l
\end{array}
$$

Um nomograma clássico útil é o apresentado por WEST, que permite o diagnóstico diferencial de praticamente todos os tipos de insuficiência respiratória (Figura 2). ${ }^{3}$ Trata-se de um nomograma de ventilação $\left(\mathrm{pCO}_{2}\right)$ /oxigenação $\left(\mathrm{pO}_{2}\right)$. Como todas as insuficiências respiratórias caracterizam se pela hipoxia, o diagnóstico diferencial é dado pelo padrão ventilatório, ou seja pela variação da $\mathrm{pCO}_{2}$.

$\mathrm{O}$ nomograma destaca as variações da $\mathrm{pCO}_{2} \mathrm{e}$ da $\mathrm{pO}_{2}$ nas diversas modalidades de insuficiência respiratória.

- Na hipoventilação pura, o aumento da $\mathrm{pCO}_{2}$ arterial é extremamente rápido, atingindo valores superiores a $80 \mathrm{mmHg}$ com vasodilatação cerebral e falência cardiorrespiratória.

- Nos casos de descompensação da doença pulmonar obstrutiva crônica (DPOC), o aumento da $\mathrm{pCO}_{2}$ 


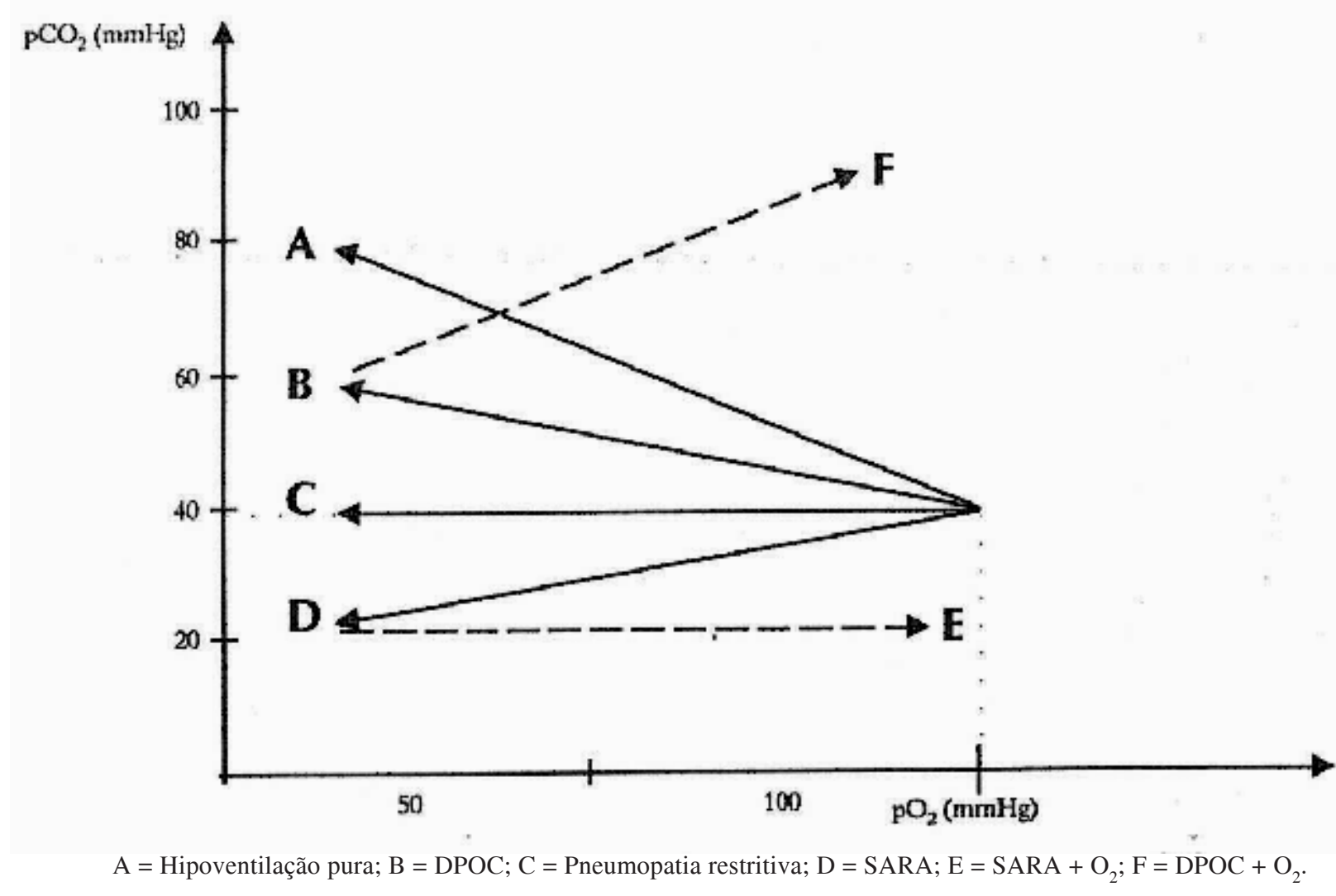

Figura 2: Nomograma $\mathrm{pCO}_{2} / \mathrm{pO}_{2}$ (West). Diagnóstico diferencial das diversas modalidades de insuficiência respiratória.

arterial é mais lento, dificilmente atingindo valores superiores a 75 a $77 \mathrm{mmHg}$.

- Nas pneumopatias restritivas, a $\mathrm{pCO}_{2}$ permanece normal, porque existe uma compensação pela característica de hiperventilação nessas doenças.

- Na síndrome da angústia respiratória do adulto (SARA) ocorre uma queda da $\mathrm{pCO}_{2}$ arterial como uma consequiência do efeito "shunt" (áreas bem perfundidas e mal ventiladas com alta difusibilidade do $\mathrm{CO}_{2}$ ).

- Com o tratamento adequado da SARA a oxigenação pode melhorar, porém a não normalização da $\mathrm{pCO}_{2}$ pode significar que ainda persistem áreas bem perfundidas e malventiladas, mantendo ainda o efeito "shunt".

- O excesso de oxigênio oferecido a pacientes portadores de DPOC pode levar à elevação da $\mathrm{pCO}_{2}$, inclusive atingindo valores superiores a $80 \mathrm{mmHg}$, o que se deve ao fato de a ventilação destes pacientes ser controlada por quimiorreceptores aórticos e carotídeos, que respondem à hipoxia e à acidose.

\section{3- CAUSAS MAIS COMUNS DOS DISTÚRBI- OS DO EAB}

De modo simplificado as causas mais comuns dos distúrbios do EAB podem, assim, ser agrupadas de acordo com os tópicos a seguir. ${ }^{2,4}$

\section{1- Acidose Respiratória}

- Aguda: por comprometimento do sistema nervoso central (poliomielite anterior aguda, intoxicações exógenas, comas, traumas); por comprometimento anatômico ou funcional da caixa torácica (ossos e músculos).

- Crônica: por lesões pulmonares (insuficiência respiratória crônica).

\section{2- Alcalose Respiratória}

- Aguda: estimulação do centro respiratório bulbar (encefalites, emoção, febre e infecções sistêmicas, intoxicação por salicilato, hipoxemia); reflexos (choque); por estimulação de receptores torácicos (atelectasia, pneumopatias agudas). 
- Crônica: vários mecanismos (assistência ventilatória, insuficiência hepática/amônia, lesões do SNC, infecções, hipoxemia, hipertireoidismo).

\section{3- Acidose Metabólica}

\section{- Por adição de ácido forte:}

- aguda exógena (infusão de $\mathrm{NH}_{4} \mathrm{CI}$, ingestão exagerada de ácido acetilsalicílico);

- aguda endógena (acidose láctica/ácido láctíco, acidose diabética/corpos cetônicos, cetose de jejum, azotemia por insuficiência renal aguda, ácido sulfúrico, ácido fósfórico);

- crônica: azotemia/insuficiência renal crônica.

\section{- Por perda de bicarbonato:}

- aguda (diarréia);

- crônica (fístula pancreática, acidose tubular renal).

\section{4- Alcalose Metabólica}

\section{- Pór perda de ácido forte:}

- aguda (vômitos);

- crônica/perda de potássio (diarréia crônica, corticosteróides, diuréticos).

\section{- Por ganho de bicarbonato:}

- aguda exógena (infusão de bicarbonato);

- aguda endógena (estresse);

- crônica (ingestão de antiácidos de ação sistêmica/bicarbonato).

Existem situações clínicas importantes que apresentam padrões de alterações do EAB que merecem atenção pela frequiência e gravidade, entre as quais se incluem:

- Estado de choque: A regra é a ocorrência de acidose metabólica devida à má perfusão tecidual com conseqüente metabolismo celular anaeróbio. Em uma fase inicial existe a tentativa de compensação respiratória, e, em fase final, a regra é uma acidose mista.

- Parada cardíaca: Ocorre acidose metabólica e respiratória, respectivamente pela parada brusca de oxigenação tecidual e da ventilação pulmonar. Nestes casos os pacientes devem ser hiperventilados, pois o tratamento da acidose é feito com o bicarbonato de sódio que é doador de $\mathrm{CO}_{2}$.
- Circulação extracorpórea: (CEC) Por melhor que seja a sua técnica, vários são os mecanismos que levam a uma inadequada perfusão tecidual (hipofluxo, hipotermia, hiperatividade simpática e ausência de fluxo pulsátil) com conseqüente acidose metabólica. Por outro lado o uso de oxigênio puro no oxigenador leva, muitas vezes, a uma acentuada diminuição do $\mathrm{CO}_{2}$. A alcalose metabólica pode ocorrer pelo excesso de bicarbonato administrado durante a CEC ou, no pós operatório, pela perda excessiva de potássio muito comum durante a derivação cardiopulmonar. Um outro problema digno de menção é a ocorrência de alcalose metabólica tardia (48 a 72 horas) no pós operatório. Pode ocorrer pelo metabolismo do citrato ou do lactato da solução de Ringer lactato no perfusado. Existem evidências de que esta alcalose seja mais devida ao citrato do anticoagulante do sangue politransfundido do que decorrente do uso de Ringer lactato. ${ }^{2}$

- Politransfusão sangüínea: O sangue estocado possui uma considerável carga de radicais ácidos, resultante em grande parte do acúmulo de ácidos orgânicos (ácido cítrico do anticoagulante e do ácido láctico gerado pela hipotermia da estocagern e pela hipoxia). Se ocorrer acidose metabólica deve se proceder à alcalinização, antes feita rotineiramente, e hoje conforme as necessidades mostradas por gasometria seriada. Além disso, o sangue estocado tem níveis de 2,3 difosfoglicerato (2,3 DPG) diminuídos, o que leva ao deslocamento da curva de dissociação da hemoglobina para a esquerda com aumento da sua afinidade pelo oxigênio, piorando a oxigenação periférica, e, conseqüentemente, agravando a acidose metabólica. Pode ocorrer, mais tardiamente, uma alcalose metabólica como resultado da metabolização do citrato pelo fígado.

- Alimentação parenteral: No início desta técnica de suporte nutricional, a acidose metabólica era uma grave e freqüente complicação. Isto ocorria porque alguns aminoácidos essenciais (histidina, lisina, ornitina e arginina), encontravam se nas soluções sob a forma de cloridrato. Esta complicação tornou se mais rara com as soluções de aminoácidos mais recentes que substituem o cloridrato por aspartato.

- Insuficiência renal crônica: A regra é a existência de acidose metabólica por adição de ácidos fortes (sulfúrico e fosfórico) decorrente da azotemia. Pode ocorrer ainda acidose metabólica pela subtração do bicarbonato devida à chamada acidose tubular 
renal. Este distúrbio é raro, freqüentemente associado a síndromes genéticas, e apresenta dois tipos: distal, devido à incapacidade do túbulo distal de manter gradiente máximo de $\mathrm{H}^{+}$entre a luz tubular e o sangue, e proximal, devido à deficiência de reabsorção do bicarbonato no início do néfron. Os pacientes portadores de insuficiência renal crônica, em geral, adaptam se à acidose metabólica.

- Diabetes melito: Ocorre acidose metabólica por adição de ácidos resultante de corpos cetônicos (ácidos acetoacético e hidroxibutírico). Muitas vezes o simples emprego da insulina é suficiente para o seu tratamento pela diminuição da produção de corpos cetônicos, sem a necessidade do emprego de bicarbonato.

- Afecções gastrointestinais: Nestas, as principais alterações podem ser divididas em três grupos: acidose metabólica, alcalose metabólica e perda de potássio. A acidose metabólica pode ocorrer por perda de bicarbonato nas diarréias graves e nas fístulas pancreáticas. A acidose pode ainda ocorrer em função do estado de choque, secundário, por exemplo, à trombose mesentérica, pancreatite aguda ou fistula pancreática. A alcalose metabólica pode ocorrer por perda de suco gástrico ou perda de potássio (diarréia, tumores vilosos do reto, tumores do pâncreas, fístulas biliares, uso excessivo de laxativos).

\section{4- TRATAMENTO DOS DESTÚRBIOS DO EQUILÍBRIO ÁCIDO BASE}

\section{1- Princípios Gerais}

Alguns preceitos devem ser seguidos para o adequado tratamento dos desvios do EAB, lembrando se que a gasometria é um exame subsidiário que deve ser analisado em conjunto com os dados clínicos. ${ }^{2,5}$

- Deve se sempre fazer o tratamento causal que, por si só, é suficiente em grande parte das alterações do EAB.

- O tratamento deve fundamentar se em dosagens freqüentes e seriadas dos gases e do $\mathrm{pH}$ sangüíneo.

- Não confiar de forma absoluta em qualquer resultado de laboratório.

- Não corrigir as alterações muito rapidamente.

- Normalizar inicialmente o volume sangüíneo e a perfusão tecidual.

- Não corrigir o pH, o cálcio e o potássio isoladamente.

\section{2- Tratamento da Acidose Respiratória}

Oxigenação e ventilação adequadas: manutenção das vias aéreas livres, correção da hipoxia e/ou hipercapnia, remoção de secreções e tratamento das infecções respiratórias quando presentes. Um erro freqüente é a administração de bicarbonato de sódio com base apenas no valor do $\mathrm{pH}$.

\section{3- Tratamento da Alcalose Respiratória}

O objetivo é combater a hiperventilação com sedação de pacientes ansiosos e histéricos. É um distúrbio freqüente em pacientes submetidos à assistência respiratória, principalmente se curarizados. A diminuição da ventilação pode ser tentada pelo aumento do espaço morto, diminuição do volume corrente e pela utilização da ventilação mecânica intermitente (IMV), que tem por característica a normalização da $\mathrm{pCO}_{2}$, uma vez que permite a respiração espontânea intercalada pelo auxílio do respirador.

\section{4- Tratamento da Síndrome da Angústia Res- piratória do Adulto (SARA)}

A SARA é um problema de "barreira alveolocapilar" causado pela presença de edema pulmonar intersticial. Caracteriza se por diminuição da capacidade residual funcional, alteração da relação ventilação/perfusão (V/Q) e aumento do "shunt" pulmonar (Qs/Qt).

Seu tratamento consiste basicamente na instalação de assistência respiratória (IMV + PEEP), restrição hídrica, diuréticos, processos dialíticos, metilprednisolona (?), albumina humana, antibióticos; a albumina deve ser usada na fase inicial para aumentar a pressão coloidosmótica e ajudar a diminuir o edema pulmonar intersticial, mas na fase avançada pode extravasar para o alvéolo, contribuindo para a formação de membrana hialina. A utilização do nitroprussiato de sódio, na tentativa de reduzir a hipertensão pulmonar, pode aumentar o desvio arteriovenoso se diminuir muito a pressão arterial sistêmica. A recente utilização de óxido nítrico inalado para reduzir a hipertensão pulmonar de modo seletivo é promissora, mas ainda controversa, havendo mais evidências de que não muda a evolução da SARA. Os valores da $\mathrm{pO}_{2}$ e da $\mathrm{pCO}_{2}$ somente voltarão à normalidade com a regressão da situação do "shunt" pulmonar aumentado. 


\section{5- Tratamento da Acidose Metabólica}

Utiliza se o bicarbonato de sódio de acordo com a fórmula de Astrup Siggaard Andersen:

$$
\frac{0,03 \times \mathrm{kg} \times \mathrm{BE}}{2 \mathrm{ou} 3}
$$

O tratamento da acidose metabólica pode ser feito por estimativa, caso não se disponha dos dados gasométricos, utilizando se, em média, $2 \mathrm{mEq} / \mathrm{kg}$ de bicarbonato de sódio como dosagem inicial, seguida por doses adicionais criteriosas. Erro comum é o emprego dessa solução alcalina em excesso, que pode aumentar muito a osmolaridade do sangue, dificultando as trocas celulares, ou, ainda, induzir arritmias cardíacas pela alcalose metabólica. O bicarbonato de sódio é, universalmente, o elemento utilizado para tratar a acidose metabólica na parada cardíaca. Tem sido estudada a utilização do tampão TRIS (THAM), que teria a vantagem de não ser doador de gás carbônico e não necessitar de hiperventilação, além de sua provável ação superior nas correções da acidose intracelular. Como não se observa a utilização rotineira do tampão TRIS, pode se inferir que, em termos de custo beneficio, este tampão pode não ter grandes vantagens sobre o uso do bicarbonato de sódio.

\section{6- Tratamento da Alcalose Metabólica}

O tratamento pode envolver reposição de cloretos, acetazolamida, cloridrato de arginina, HLCI $(0,1 \mathrm{~N}), \mathrm{NH}_{4} \mathrm{CI}($ ?). A utilização de cloridrato de arginina é empírica (7,5g/12h), uma vez que só a sua fração catabolizada é acidificante. Conseguem se reversões parciais da alcalose metabólica, que se recupera algumas horas depois. Utiliza se mais a reposição de cloreto de potássio em associação com a acetazolamida, a qual, inibindo a anidrase carbônica, aumenta a excreção urinária de bicarbonato. A experiência com HCL é pequena e o $\mathrm{NH}_{4} \mathrm{CL}$ é neurotóxico. ${ }^{2,6,7}$

\section{5- EFEITOS DELETÉRIOS DOS DISTÚRBI- OS DO EQUILÍBRIO ÁCIDO BASE}

Tanto a acidose como a alcalose apresentam uma série de efeitos indesejáveis. ${ }^{2,8}$ Os efeitos indesejáveis da acidose aguda estão apresentados no Quadro 2. Os efeitos deletérios da alcalose aguda estão representados no Quadro 3.
A alcalose metabólica, cuja importância clínica costuma ser relegada a um plano secundário, merece um lugar de destaque entre os possíveis distúrbios da homeostase do paciente gravemente enfermo e dos submetidos a grandes cirurgias. Além de aumentar a afinidade da $\mathrm{Hb}$ pelo $\mathrm{O}_{2}$, tem se dado importância à alcalose metabólica como causa de arritmias cardíacas, principalmente supraventriculares que não apresentam boa resposta aos antiarrítmicos convencionais, admitindo se, inclusive, a ocorrência de morte súbita e sua correlação com aumento da mortalidade em pacientes graves.

A Figura 3 apresenta um resumo das possibilidades diagnósticas e terapêuticas dos distúrbios do EAB. Este quadro, associado ao nomograma de West $\left(\mathrm{pCO}_{2} / \mathrm{pO}_{2}\right)$, resume grande parte dos conhecimentos utilizados na prática clínica diária.

Quadro 2: Efeitos deletérios da acidose aguda.

- Sobrecarga respiratória

- Anorexia, náuseas, vômitos e alterações neurológicas

- Hiperpotassemia

- Diminuição da responsividade às catecolaminas e depressão da contratilidade miocárdica

- Vasoconstrição renal e oligúria

- Resistência à ação da insulina

Quadro 3: Efeitos deletérios da alcalose aguda.

- Hipocalcemia por diminuição do cálcio ionizável

- Hipopotassemia com aumento da perda urinária de $\mathrm{K}^{+}$

- Efeito da alteração da relação $\mathrm{K}^{+}$íntracelular/K $\mathrm{K}^{+}$ extracelular no míocárdio com suscetibilidade a arritmias

- Aumento da afinidade da $\mathrm{Hb}$ pelo $\mathrm{O}_{2}$ (desvio da curva de dissociação para a esquerda), com hipoxía tecidual

- Acidose paradoxal do líquor com piora das condições neurológicas 


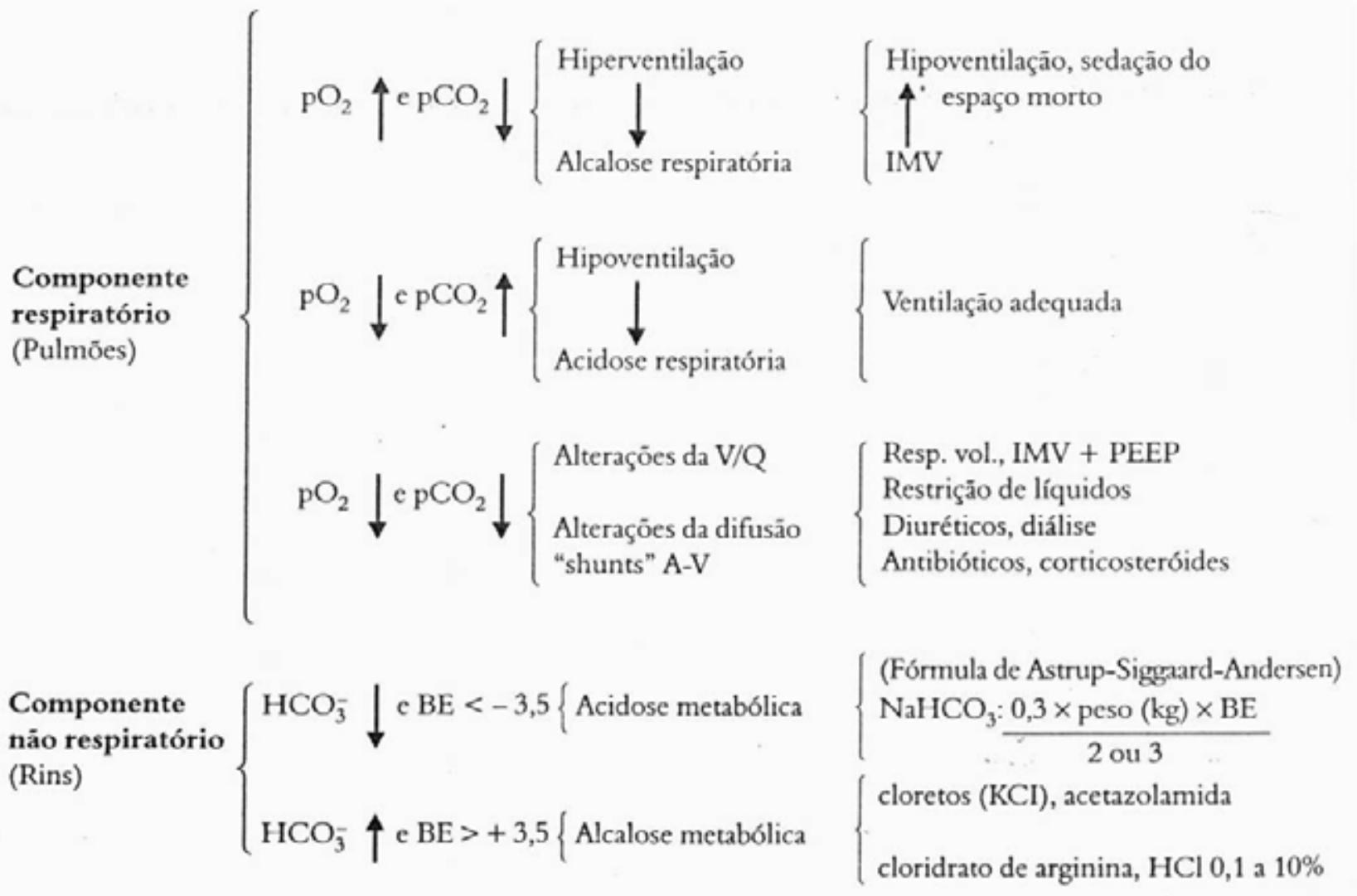

V/Q = Relação ventilação/perfusão; PEEP = Pressão expiratória positiva final; IMV = Ventilação mecânica intermitente.

Figura 3: Resumo das possibilidades diagnósticas e terapêuticas dos distúrbios do equilíbrio ácido base.

\section{6- EXERCÍCIOS GASOMÉTRICOS}

A gasometria é um exame complementar que deve ser analisado em associação com o quadro clí- nico apresentado pelo paciente.

Os diagnósticos dos casos apresentados no Quadro 4 são orientados pelos dados da Figura 3.

\section{Quadro 4: Exercício de interpretação de resultados da gasometria.}

CASO 1: $\mathrm{pH}=7,30 ; \mathrm{pO}_{2}=86 ; \mathrm{pCO}_{2}=39 ; \mathrm{HCO}_{\overline{3}}^{-}=18,12 ; \mathrm{BE}=-7,5$

Diagnóstico: ausência de alterações respiratórias e acidose metabólica leve.

Conduta: acidose com BE até 8 sem sobrecarga respiratória não necessita de tratamento, ocorrendo compensação pelo próprio organismo. Observar que o valor do $\mathrm{pH}$ está abaixo do normal, o que é freqüente na acidose do jejum pré-operatório.

CASO 2: $\mathrm{pH}=7,42 ; \mathrm{pO}_{2}=99 ; \mathrm{pCO}_{2}=21,39 ; \mathrm{HCO}_{3}=18,12 ; \mathrm{BE}=8,0$

Diagnóstico: acidose metabólica compensada também por mecanismo respiratório.

Conduta: esta é uma resposta natural do organismo, ou seja, a hiperventilação para eliminação indireta do $\mathrm{H}^{+}$. Apesar de o BE ser semelhante ao do Caso 1, neste caso recomenda se tratar a acidose com $\mathrm{HCO}_{3}^{-}$, pois esta deve 
ser importante a ponto de desencadear os mecanismos respiratórios de compensação. A oferta de nessa situação visa evitar a sobrecarga respiratória, indesejável no paciente criticamente doente. Esta conduta não é unânime; há quem se limita ao tratamento causal quando o pH está normal.

CASO 3: $\mathrm{pH}=7,61 ; \mathrm{pO}_{2}=91 ; \mathrm{pCO}_{2}=41 ; \mathrm{HCO}_{\overline{3}}=39,81 ; \mathrm{BE}=+18$

Diagnóstico: ausência de alterações respiratórias e alcalose metabólica.

Conduta: uma boa parte das alcaloses metabólicas está associada à hipopotassernia e/ ou hipocloremia, portanto, estes íons devem ser dosados no plasma. Caso estejam normais, optar por outro agente acidificante: HCL 0,1 a 0,2 $\mathrm{N}$, cloridrato de arginina. Lembrar que a acetazolamida é de grande utilidade no tratamento das alcaloses de adição e que o cloreto de amônio tem seu uso limitado pela neurotoxidade.

CASO 4: $\mathrm{pH}=7,29 ; \mathrm{pO}_{2}=60 ; \mathrm{pCO}_{2}=57 ; \mathrm{HCO}_{\overline{3}}^{-}=26,5 ; \mathrm{BE}=1$

Diagnóstico: acidose respiratória sem alterações metabólicas.

Conduta: esta gasometria pode ser perfeitamente aceitável para um paciente portador de doença pulmonar obstrutiva crônica. Caso não se trate de um paciente enfisematoso, deve se buscar a causa da hipoventilação e tratá la por meio de ventilação adequada.

Caso 5: $\mathrm{pH}=7,45 ; \mathrm{pO}_{2}=55 ; \mathrm{pCO}_{2}=61 ; \mathrm{HCO}_{\overline{3}}^{-}=26,5 ; \mathrm{BE}=+19$

Diagnóstico: acidose respiratória e alcalose metabólica.

Conduta: em pacientes não portadores de doença pulmonar obstrutiva crônica, devem se tratar ambos os desvios do equilíbrio ácido base. Comumente interpreta se o presente quadro gasométrico como acidose respiratória compensada por alcalose metabólica, o que leva à conduta inadequada. Costumam se tratar os dois distúrbios, pois sabe se que os mecanismos naturais de defesa contra a acidose são muito mais eficientes do que os mecanismos de defesa contra a alcalose. Por outro lado, se o paciente for um enfisematoso pode se interpretar a alcalose metabólica como uma conseqüência da hipercapnia crônica, devendo se tratá la com o emprego de acetazolamida.

CASO 6: $\mathrm{pH}=7,63 ; \mathrm{pO}_{2}=100 ; \mathrm{pCO}_{2}=22 ; \mathrm{HCO}_{3}^{-}=22,36 ; \mathrm{BE}+4,5$

Diagnóstico: alcalose respiratória e alcalose metabólica leve.

Conduta: tratar a alcalose respiratória. Este é um distúrbio comum que ocorre com o uso de respiradores mecânicos e, no caso, poder-se-á diminuir a freqüência respiratória ou aumentar o espaço morto. Uma vez que a $\mathrm{pO}_{2}$ se apresenta em níveis normais, pode não ser conveniente diminuir o volume corrente. Na hiperventilação psicogênica deve se promover a sedação do paciente ou, se possível, fazê lo respirar em saco fechado.

CASO 7: $\mathrm{pH}=7,30 ; \mathrm{pO} 2=55 ; \mathrm{pCO} 2=18 ; \quad \mathrm{HCO}_{\overline{3}}=20,2 ; \mathrm{BE}=4,0$.

Diagnóstico: sempre que houver hipoxia com hipocapnia deve se pensar em alterações pulmonares funcionais (alteração da relação ventilação perfusão, da difusão alveolocapilar, "shunts" arteriovenosos intrapulmonares). Na prática médica, até prova em contrário, trata se de insuficiência respiratória aguda do adulto conseqüente a edema pulmonar intersticial.

Conduta: procurar tratar a doença de base, assistência respiraatória com respirador volumétrico e IMV + PEEP, metilprednisolona, furosemida, diálise e albumina humana quando houver hipoproteinemia nas primeiras fases da SARA.

Évora PRB, Garcia LV. Acid-base balance. Medicina (Ribeirão Preto) 2008; 41 (3): 301-11.

ABSTRACT: A profound knowledge about variations in the acid-base balance is of utmost importance for both the correct diagnosis and the treatment of possible abnormalities. Here, the causes and consequences, as well as potential treatments of both respiratory and metabolic alkalosis and acidosis are discussed.

keywords: Acid-Base Equilibrium. Alkalosis. Acidosis. 


\section{BIBLIOGRAFIA RECOMENDADA}

1 - Cohen JJ, Kassirer JP Metabolismo ácido básico. In: Maxwell \& Kleeman. Clínica das Alterações Eletrolíticas. 32 ed., Rio de Janeiro, Guanabara Koogan, 1981; p. 132-67.

2 - Évora PRB, Reis CL, Ferez MA, Conte DA, Garcia LV Distúrbios do equilíbrio hidroeletrolítico e do equilíbrio ácido básico. Uma revisão prática. Medicina (Ribeirão Preto) 32: 451-69, 1999.

3 - West JB. Insuficiência respiratória. In: WEST JB. Fisiopatologia Pulmonar Moderna. $2^{\mathrm{a}}$ ed., São Paulo, Manole, 1986; p. 157-70.

4 - Birolini D. Nomenclatura e classificação dos distúrbios ácido básicos. In: Faintuch J, Birolinl D, Machado MC. Equilíbrio Ácido básico na Prática Clínica. São Paulo, Manole, 1975; p. 29-49.
5 - Wilson RF Alterações ácido básicas no choque clínico. In Schumer W, Nihus LM. Tratamento do Choque. São Paulo, Manole, 1976; p. 44-63.

6 - Évora PRB, Reis CL, Ribeiro P JF, Brasil JCE, Otaviano AG, Silva JRP, Margarido EA. Alcalose metabólica. Tratamento com solução de aminoácidos contendo cloridrato, de arginina. Rev. paul. med. 1981; 97: 25-8.

7 - Évora PRB. Treatment of the hypocloremic metabolic alkalosis by rectal infusion of chloride. Crit care med. 1985; 13 : 874.

8 - Faintuch J. Conduta prática nas acidoses e alcaloses. In: Faintuch J, Birolini D, Machado MC Equilíbrio Ácido básico na Prática Clínica. São Paulo, Manole, 1975; p. 161-5.

Recebido para publicação em 20/08/2008

Aprovado para publicação em 30/09/2008 